\title{
Determination and elimination of rotten grape aroma by filter layers with selective adsorbers
}

\author{
Reinhard Eder and Stefan Nauer \\ Höhere Bundeslehranstalt und Bundesamt für Wein- und Obstbau, Wiener Straße 74, 3400 Klosterneuburg, Österreich
}

\begin{abstract}
The most important mould associated substances like geosmine, 2-methylisoborneol, 1-octen-3-ol in wines of different grape varieties from Austria and also from other foreign countries were analysed by a HS-SPME GC-MS method. These substances were also determined in healthy and rotten grapes. All together, 391 wine-samples and 69 grape samples were examined.

The study of rotten and healthy grapes found out a level of geosmin up to $20 \mathrm{ng} / \mathrm{L}, 1$-octene-3-ol concentration up to $700 \mu \mathrm{g} / \mathrm{L}$ and 2-heptanol level up to $9 \mu \mathrm{g} / \mathrm{L}$. The geosmin concentration of the 391 analyzed wines extended from 1-44 ng/L. The detected level of 1-octene-3-ol ranged from $1 \mu \mathrm{g} / \mathrm{L}$ to $23 \mu \mathrm{g} / \mathrm{L}$. The determined concentration of 2-heptanol ranged from $1 \mu \mathrm{g} / \mathrm{L}$ up to $38 \mu \mathrm{g} / \mathrm{L}$. The test with the filter layer Fibrafix TX-R was performed with nine layers á $20 \times 20 \mathrm{~cm}\left(3.6 \mathrm{~m}^{2}\right)$ and a quantity of 625 liters wine. The filtering achieved a significant reduction of the start concentration of $60 \mathrm{ng} / \mathrm{L}$ geosmin down to only $6 \mathrm{ng} / \mathrm{L}$ (average value of $6001)$.
\end{abstract}

\section{Aromen gefaulter Trauben}

Die Anwesenheit von einigen Fehlaromen (off-flavours) in Trauben oder Weinen ist eine Folge der Entwicklung von pflanzenpathogenen Pilzen auf Trauben, die in der Literatur schon seit längerer Zeit beschrieben sind. (Semichon, 1905; Ribereau-Gayon und Peynaud, 1964). Auffällig ist aber, dass während der letzten zehn Jahre das Auftreten aromatischer Defekte mit pilzigem, schimmeligem, medizinischem oder erdigem Charakter verstärkt festgestellt wurde.

Folgende Verbindungen gelten als Leitsubstanzen für dieses Schimmel- bzw. Muffaroma im Wein und Trauben: Geosmin, 1-Octen-3-ol, 2-Methylisoborneol, 1-Octen3-on, 1-Nonen-3-on. Geosmin, aus dem Griechschen übersetzt, bedeutet Erdgeruch $($ geo $=$ Erde osme $=$ der Geruch) [Gerber und Lechevalier, 1965]. Es handelt sich dabei um eine organische Verbindung, die durch ihren eindeutigen und unverwechselbaren erdigen OffFlavour, welcher vermutlich durch verschiedene Bakterien und Pilze produziert wird, erkennbar ist. Diese nach Erde riechende Verbindung wurde zum ersten Mal 1891 identifiziert, 1965 von Gerber und Lechevalier aus verschieden Actinomycten Arten isoliert und im Jahre 1968 erstmals synthetisiert [Cotton, 2008]. Laut Amon et al. (1989) wurde Geosmin erstmals 1989 in Wein und im Jahr 2000 im Most (Darriet et al., 2000) nachgewiesen. Dieser Nachweis im Most verdeutlichte, dass nicht nur die Mikroorganismen im Fass und bei der Gärung die verantwortlichen Produzenten von Geosmin sein können. Darriet et al. (2001) erbrachten den Beweis, dass von Trauben isolierte Mikroorganismen (Streptomyces sp. und Penicillium sp.,) Geosmin produzieren können. LaGuerche et al. (2007) konzentrierten sich darauf, warum mit Botrytis infizierte Weintrauben nicht mit Geosmin kontaminiert wurden. Sie stellten fest, dass für die Geosminproduktion sowohl bestimmte BotrytisPilze wie auch Penicillium expansum vorhanden sein müssen. Von Wnuk et al. (1983) wird 1-Octen-3ol als die Hauptaromakomponente von Pilzen und hauptverantwortlich für den typischen Pilzgeruch genannt. Lee und Simpson (1993) stellten fest, dass 1-Octen-3ol neben dem Pilzgeruch auch einen schimmelähnlichen Geschmack in Wein verursachen kann. Auch Simpson (1990) fand, dass Octenol in unsauberen Weinen einen Pilzgeruch (19\%) verursachen kann.

Medsker et al. (1969) und Gerber (1969) identifizierten bei Tests von natürlichem Wasser eine mufffige riechende Verbindung, nämlich 2-Methylisoborneol (2-MIB). 2 Paterson et al. (2007) beschreiben, dass Methylisoborneol ein Metabolit von B. cinerea, Penicillium und Streptomyces ist, und einen ähnlichen Geruch wie Geosmin hat. Bei Darriet et al. (2007) ist $\mathrm{zu}$ lesen, dass Botrytis fähig ist, 2-Methylisoborneol mit einem erdigen und kampferartigen Geruch zu produzieren. Außerdem weisen auch Paterson et al. (2007), darauf hin, dass Methylisoborneol in faulen Weintrauben der Sorte Merlot nachgewiesen wurde. Schließlich wurde die Verbindung dann in Wein mit erheblicher Korkbelastung wahrgenommen; dies ist eine wichtige Beobachtung für Korkerzeuger wie zum Beispiel Portugal (Paterson et al., 2007). Der Geruchschwellenwert im Wein für 2-Methylisoborneol liegt bei $30 \mathrm{ng} / \mathrm{L}$ (Amon et al., 1989).

\section{Maßnahmen zur vermeidung und reduzierung vonSchimmeltönen}

Bisher übliche Maßnahmen um Schimmelton vorbeugend zu begegnen:

- Effizienter Pflanzenschutz im Weinberg zur Vermeidung von Infektionen 
- Aussortierung fauler, pilzbefallener Trauben

- Sauberkeit im Keller (Reinigung und Desinfektion)

- Kohlebehandlung im Most-Stadium.

Neben diesen praktikablen Maßnahmen existieren noch weitere Möglichkeiten die Schimmelmufftöne, allen voran die Verbindung Geosmin zu reduzieren. Lisanti et al. (2009) fanden dass die Behandlung mit Traubenkernöl eine effiziente Verringerung des Erdgeruches in Rot- und Weißwein bewirkt (ca. 70\%).

Übliche Schönungsmittel wir Gelatine, Albumine und Kaseinate. Silikate und Bentonit zeigen nur eine geringe, unspezifische Wirkung gegenüber schimmelassozierten Aromen. Ähnliche, nicht zielgerichtete Effekte lassen sich auch mit der sogenannten Tiefenfiltration erzielen.

Bei der Schönung mit Aktivkohle handelt es sich um das am stärksten adsorptiv wirkende Weinbehandlungsmittel. Farkaš et al. (1988) erwähnt, dass Aktivkohle am häufigsten für die Korrektur von verschimmelten Weinen oder Weine mit einem schlechten Geschmack eingesetzt wird. Falls der Wein mit starken Adsorptionsmitteln, wie beispielsweise mit Aktivkohle behandelt wird, können die unerwünschten Aromakomponenten (TCA, muffigen Ton usw.) in den meisten Fällen entfernt werden.

Neben Aktivkohle sind auch Polyethylen und die Milchprodukte in der Behandlung von muffigen Wein relativ erfolgreich. Vor einigen Jahren wurde von der Schweizer Firma Filtrox Tiefenfilterschichten mit eingebetteten Zeoliten entwickelt, die Korkgeschmack entfernen können. Die Tiefenfilterschichten FIBRAFIX ${ }^{\circledR}$ TX-R bietet eine wirksame Methode zur Entfernung von TCA (2, 4, 6-Trichloranisol) aus Wein und anderen Getränken. Im Gegensatz zu anderen Methoden der TCA-Entfernung wird der ursprüngliche Geruch und Geschmack durch die TX-R Schichten nicht zerstört. Die Eignung des Produktes Fibrafix TX-R zur Entfernung von TCA und TBA aus Wein wurden von JUNG et al. (2008) beschrieben.

$\mathrm{Zu}$ ähnlichen und weiter reichenden Erkenntnissen über diese Spezialfilterschichten gelangten auch EDER et al. (2008).

\section{Inhalt der präsentation}

- Vorkommen von Geosmin und weiteren schimmelassoziierten Verbindungen wie 1-Octen-3-ol, 2-Methylisoborneol, 2-Octanol, 3-Octanol in verschiedenen Trauben bzw. Weinen unter Berücksichtigung des Gesundheitszustandes und der Sorte geklärt werden. Quantifizierung mittels SPME- GC- MS Technik.

- Bestimmung der Kapazität einer Tiefenfilterschicht mit Zeolite (FibrafixTX-R, Fa. Filtrox) zur Entfernung schimmelassoziierter im großtechnischen Maßstab.

\section{Material und methoden}

Es wurden 69 Proben von gesunden und geschimmelten Trauben sowie 391 Weinproben, die bei der amtlichen Verkostung als korkig, muffig oder schimmelig beanstandet wurden, analysiert.

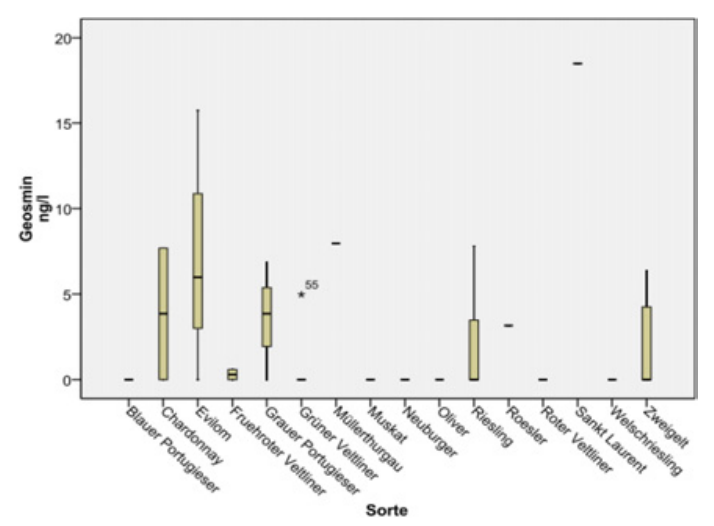

Abb. 1. Geosmingehalte in gefaulten Trauben.

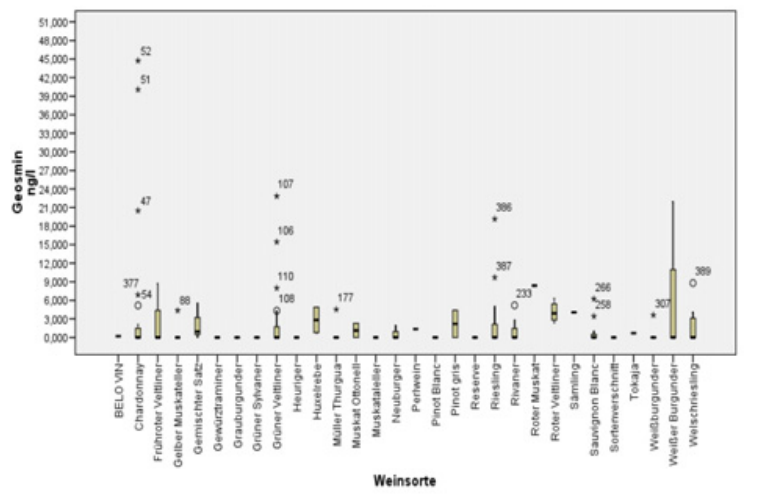

Abb. 2. Geosmingehalte in Weißweinen.

Vor der Analyse wurden die Zielsubstanzen mittels Festphasenmikroextraktion (SPME mit $100 \mu \mathrm{m}$ Polydimethylsiloxan-PDMS) aufgereinigt. Die Bestimmung erfolgte mittels Gaschromatographie gekoppelt mit Massenspektrometer (GC-MS).

\section{Ergebnisse}

Die Geosmingehalt in den 69 Proben von geschimmelten Trauben (Abb. 1) zeigen Medianwerte im Bereich bis zu $6 \mathrm{ng} / \mathrm{l}$ und einzelne Höchstwerte bis $18 \mathrm{ng} / \mathrm{l}$.

Von den Ergebnissen der Weinproben werden beispielshalt die Ergebnisse der Weißweine dargestellt (Abb. 2), aus der man ersieht, dass nur in wenigen Weinen Geosmin gefunden wird. Diese Proben waren aber zum Teil erheblich mit Werten bis zu $45 \mathrm{ng} / \mathrm{l}$ kontaminiert.

Den für die Testung der Tiefenfilterschichten mit Zeolith Fibrafix TX-R verwendeten Weißwein wurden vor der Filtration $60 \mathrm{ng} / \mathrm{L}$ Geosmin zugesetzt. Während der Filtration wurde in Abständen von $25 \mathrm{~L}$ Fraktionen gezogen und deren Konzentration an Geosmin mit HSSPME GC analysiert.

$\mathrm{Zu}$ Beginn der Filtration wurde die zugesetzte Geosminkonzentration zu praktisch $100 \%$ reduzierten. Erst nach rund $150 \mathrm{~L}$ begann ein äußerst langsamer Anstieg an Geosmin in den Fraktionen, augenscheinlich wo die ersten aktiven Stellen der Filterschichten abgebunden wurden. Trotzdem schreitet die Zunahme von Geosmin sehr zögerlich voran, sodass nach der gesamten Filtrationsmenge von 625 Liter nur eine Geosminkonzentration von $15 \mathrm{ng} / \mathrm{L}$ erreicht wurde. Dies bedeutet am Ende der Filtration eine Reduzierung vom 
ursprünglichen Startwert um 75\%. Es konnte damit deutlich bestätigt werden, dass dieser Spezialfilter nicht nur zur ursprünglichen Entfernung von TCA geeignet sind sondern auch Geosmin in größerem Maßstab entfernen können. Dies bestätigen die publizierten Versuche im Labormaßstab von Eder et al. (2008).

\section{Literatur}

J.M, Amon., J.M. Vandepeer and R.F. Simpson : Australian and New Zealand Wine Industry Journal 4, 62-69 (1989)

P. Darriet, M. Pons; S. Lamy and D. Dubourdieu: J. Agric. Food. Chem. 10 (2000). pp 4835-4838

R. Eder,. M. Hütterer, G. Weingart, W. Brandes: Mitt. Klosterneuburg. 58/2 (2008). 12-14

J. Farkas, und Z. Procházka: Technology and biochemistry of wine Gordon and Breach Science Publ., New York ISBN:2-88-124-069-0 (1988). 744

N.N. Gerber, and H.A. Lechevalier: Appl. Environ Microb (1965). 935-938
R. Jung, V. Schaefer, M. Chistmann, M..Hey und S. Fischer and D. Rauhut: Mitt. Klosterneuburg. 2 (2008). $58-67$

S. LaGuerche, L., Senneville; D., Blancard and P. Darriet: Anton Leeuw Int. 92(3) (2007). 331-341

H. Lee, and R.F. Simpson: In: G.H. Fleet, Editor, Wine Microbiology and Biotechnology, Harwood Academic Publisher, Chur, Switzerland (1993), pp. 353-372

T. Lisanti, P. Piombino, A. Gambuti, A. Genovese, and L. Moio:. Bulletin de 1'OIV vol. 82, no. 935-937 ISSN 0029-7127 (2009). pp. 71-82

L. Medsker L., D. Jenkins; J.F. Thomas and C. Koch Environ Sci Technol, 3 (5), (1969). 476-477

J. E. Ribéreau-Gayon, et E. Peynaud: Traité d'Oenologie Tome 1 : Maturation du raisin, fermentation alcoolique, vinification., Béranger et Dunod éditeurs, Paris (1964)

Semichon: Traité des maladies des vins Masson éditeur, Paris (1905)

S. Wnuk, S. Kinastowski and E. Kaminski: Nahrung 27(5): (1983). 479-486 